\title{
Interactions between shallow and deep convection under a finite departure from convective quasi-equilibrium
}

Article

Published Version

Yano, J.-I. and Plant, R. (2012) Interactions between shallow and deep convection under a finite departure from convective quasi-equilibrium. Journal of the Atmospheric Sciences. ISSN 1520-0469 doi: https://doi.org/10.1175/JAS-D-12-0108.1 Available at https://centaur.reading.ac.uk/28887/

It is advisable to refer to the publisher's version if you intend to cite from the work. See Guidance on citing.

To link to this article DOI: http://dx.doi.org/10.1175/JAS-D-12-0108.1

Publisher: American Meteorological Society

All outputs in CentAUR are protected by Intellectual Property Rights law, including copyright law. Copyright and IPR is retained by the creators or other copyright holders. Terms and conditions for use of this material are defined in the End User Agreement.

www.reading.ac.uk/centaur

\section{CentAUR}


Central Archive at the University of Reading

Reading's research outputs online 


\title{
Interactions between Shallow and Deep Convection under a Finite Departure from Convective Quasi Equilibrium
}

\author{
JUN-ICHI YANO \\ GAME/CNRM, Météo-France and CNRS, Toulouse, France \\ ROBERT PLANT \\ Department of Meteorology, University of Reading, Reading, United Kingdom
}

(Manuscript received 5 April 2012, in final form 20 July 2012)

\begin{abstract}
The present paper presents a simple theory for the transformation of nonprecipitating, shallow convection into precipitating, deep convective clouds. To make the pertinent point a much idealized system is considered, consisting only of shallow and deep convection without large-scale forcing. The transformation is described by an explicit coupling between these two types of convection. Shallow convection moistens and cools the atmosphere, whereas deep convection dries and warms the atmosphere, leading to destabilization and stabilization, respectively. Consequently, in their own stand-alone modes, shallow convection perpetually grows, whereas deep convection simply damps: the former never reaches equilibrium, and the latter is never spontaneously generated. Coupling the modes together is the only way to reconcile these undesirable separate tendencies, so that the convective system as a whole can remain in a stable periodic state under this idealized setting. Such coupling is a key missing element in current global atmospheric models. The energy cycle description used herein is fully consistent with the original formulation by Arakawa and Schubert, and is suitable for direct implementation into models using a mass flux parameterization. The coupling would alleviate current problems with the representation of these two types of convection in numerical models. The present theory also provides a pertinent framework for analyzing large-eddy simulations and cloud-resolving modeling.
\end{abstract}

\section{Introduction}

The representation of convective cloud is a key element for successful synoptic weather forecasts and climate projection (cf. Randall et al. 2007). The transformation of nonprecipitating, stratocumulus-topped boundary layers into trade wind convection, and then into precipitating, deep convective clouds is an especially challenging issue, with extensive efforts underway aimed at both process understanding and modeling [e.g., Wu et al. (2009) and references therein]. In the present paper, by shallow convection, we loosely refer to both stratocumulus and trade wind convection, but with more emphasis on the latter. In current global models, exclusive parameterizations have been developed for shallow and deep convection: thus, at the moment of transformation,

Corresponding author address: Jun-Ichi Yano, CNRM, MétéoFrance, 42 Ave. Gaspard Coriolis, 31057 Toulouse CEDEX 1, France.

E-mail: jun-ichi.yano@zmaw.de shallow convection is simply turned off, and deep convection is turned on [as in Tiedtke (1989), e.g.; see also Plant (2010)].

By presenting a simple theory for this transformation, the present paper shows the importance of the coupling of shallow and deep convection, which is key to successful simulations of the transformation process. The theory is presented in terms of an energy cycle of convective systems established by Arakawa and Schubert (1974) under their mass flux formulation. Pan and Randall (1998) and Yano and Plant (2012) have further explored this formulation. Since this energy cycle formulation can be formally derived from a general form of the mass flux parameterization, it is both robust and general. Although the present paper analyzes this energy cycle system under an extremely simplified setting, the formulation itself is valid even under fully realistic, operational settings. For this reason, the present study leads to an important practical implication.

To present our theory in as lucid a manner as possible, a very idealized setting is considered consisting only of 
shallow and deep convection without large-scale forcing. Such a situation never arises in nature. A steady subcloud-layer state is also assumed in order to focus our attention on the interactions of convection with its environment. Clearly, the theoretical setting is highly idealistic. However, such a drastic idealization serves the purpose of elucidating the contrasting nature of shallow and deep convection. Furthermore, the general applicability of the energy cycle formulation adopted here hardly diminishes under all these idealizations.

Note particularly that although stratocumulus clouds are often driven by nocturnal cloud-top radiative cooling (cf. Moeng 1998; Bretherton et al. 2004), this process does not play a role in the present analysis. The focus of the study is exclusively on the impact of convection on the environment in changing the stability. Radiative cooling is required to close the heat budget for convective elements in stratocumulus clouds but does not enter here once we implicitly adopt the notion of a steadystate convective plume as assumed in standard mass flux convection parameterization.

Moist atmospheric convection is characterized by a wide range of convective clouds, even within a homogeneous large-scale environment (cf. Stevens 2005). Spectral models may be necessary in order to account for the full range of clouds (Arakawa and Schubert 1974). However, it has often proved convenient in practice to consider convective clouds as belonging to one of two major types: shallow and deep. Shallow convection has a cloud top that is close to the top of the planetary boundary layer, whereas the cloud tops for deep convection extend into the free troposphere, and can even penetrate the tropopause. Notwithstanding their names, these two basic categories of convection are more fundamentally distinguished as being nonprecipitating (or perhaps only weakly precipitating) and precipitating (often strongly) states. This distinction is the fundamental one because it leads to contrasting forms of interaction between the convection and the atmospheric environment. The contrast can best be elucidated by comparing their respective energy cycles, as presented in the next section.

\section{Theory}

We start our investigation by establishing the role of the cloud work function originally introduced by Arakawa and Schubert (1974) in the context of the convective energy cycle. Recall that Arakawa and Schubert's cloud work function $A$ is defined by

$$
A=\int_{z_{B}}^{z_{T}} \eta b d z .
$$

Here, $\eta$ is a normalized convective mass flux defined immediately below, and $b$ is the convective buoyancy. The limits of the integral range from the cloud base $z_{B}$, where ascending air becomes saturated, to the cloud top $z_{T}$, where the buoyancy vanishes.

The physical meaning of the cloud work function may be best understood in the following manner. By definition convection is a type of fluid motion that is driven by $b$. A simple energy integral shows that the kinetic energy for convective motion is locally generated at rate $b m$ per unit volume, where $m$ is the vertical momentum of the air per unit volume (cf. Yano et al. 2005a). Convection does not occur everywhere over a large-scale domain, but it occupies a fractional area $\sigma_{c}$. Thus, the buoyancy generates convective kinetic energy $K$ in an atmospheric column of unit horizontal area at a rate given by

$$
\int_{z_{B}}^{z_{T}} M b d z=M_{B} A
$$

with $M=\sigma_{c} m$ designating the upward convective momentum per unit model volume. The quantity $M$ is known as the convective mass flux, and it can be normalized using its cloud-base value $M_{B} \equiv M\left(z_{B}\right)$, so that $M=\eta M_{B}$.

From Eq. (2), we see that $A$, defined by Eq. (1), measures the efficiency of convective kinetic energy generation produced by a unit of cloud-base mass flux. The evolution of the convective kinetic energy associated with each convective type may be described by

$$
\frac{d K}{d t}=M_{B} A-\frac{K}{\tau} .
$$

This corresponds to Eq. (132) of Arakawa and Schubert (1974). Here, we follow the assumption of Lord and Arakawa (1980; also Pan and Randall 1998; Yano and Plant 2012) that the kinetic energy dissipation can be simply characterized by a time scale $\tau$.

The generated convection, in turn, modifies $A$ with a rate proportional to $M_{B}$. Here, it is important to realize that each convective type, say, designated by subscript $j$, modifies the cloud work function for all the convective types, designated by the subscript $i$. The cloud work function is also generated by large-scale processes $F$. Thus, by adding subscripts $i$ and $j$ to all the variables, a general equation for the cloud work function is given by

$$
\frac{d A_{i}}{d t}=\sum_{j=1}^{N} \mathcal{K}_{i, j} M_{B, j}+F_{i},
$$

corresponding to Eq. (142) of Arakawa and Schubert (1974). Here, $\mathcal{K}_{i, j}$ designates a rate that the $j$ th type of convection modifies the cloud work function for $i$ th type 
of convection per unit of mass flux $M_{B, j}$ and $N$ types of convection can be considered in general. A careful derivation of this equation is presented in appendix $\mathrm{B}$ of Arakawa and Schubert (1974; see also Yano and Plant 2012).

We, again, emphasize that the energy cycle of convective systems defined by Eqs. (3) and (4) is general, in the sense that it is satisfied by more or less all the existing mass flux convection parameterizations. Here, we assume that a set of $\eta$ of convection is prescribed by given entrainment and detrainment rates. This assumption is justified when convective processes are much faster than the large-scale processes, so that each convective type is in steady state (i.e., steady plume hypothesis). In deriving Eq. (4), we furthermore assume that there are no direct interactions between different convective types, so that their mutual interactions occur only through their modifications of the environment (large-scale state), which consequently modifies the cloud work functions for the other convective types.

Recall that Arakawa and Schubert's (1974) convective quasi-equilibrium hypothesis is defined by a steady state to Eq. (4). The present study, in turn, by considering Eqs. (3) and (4) fully prognostically, examines a finite departure of convective systems from convective quasi equilibrium.

In the present paper, we take the above-mentioned energy cycle formulation, consisting of Eqs. (3) and (4), and apply a strong truncation to two modes, $N=2$, consisting of shallow and deep convection only. The modes are designated by subscripts $s$ and $d$ in the following. Also, as a further idealization, we totally neglect the contribution of the large-scale forcing by setting $F_{s}=$ $F_{d}=0$. As discussed in the introduction, the question of transformation from shallow to deep convection is thus considered in isolation in the most idealized manner.

To establish the feedback loops described by the matrix $\mathcal{K}_{i, j}$, it is important to realize that shallow and deep convection interact with the atmospheric environment in contrasting ways (Fig. 1). Note that although our theoretical formulation, being based on the mass flux formulation, is less directly applicable to stratocumulus than trade cumulus convection; nonetheless, the following arguments are equally applicable on a conceptual level to stratocumulus.

The dominant action of shallow convection arises from detrainment of cloudy air around cloud top. Typically, the detrained cloud water evaporates as it mixes with the environment, and the resulting cooling destabilizes the atmosphere. The process is schematically shown by Fig. 12 of Arakawa and Schubert (1974). In terms of the energy cycle, this process is manifest as a positive feedback that increases the cloud work functions (a)
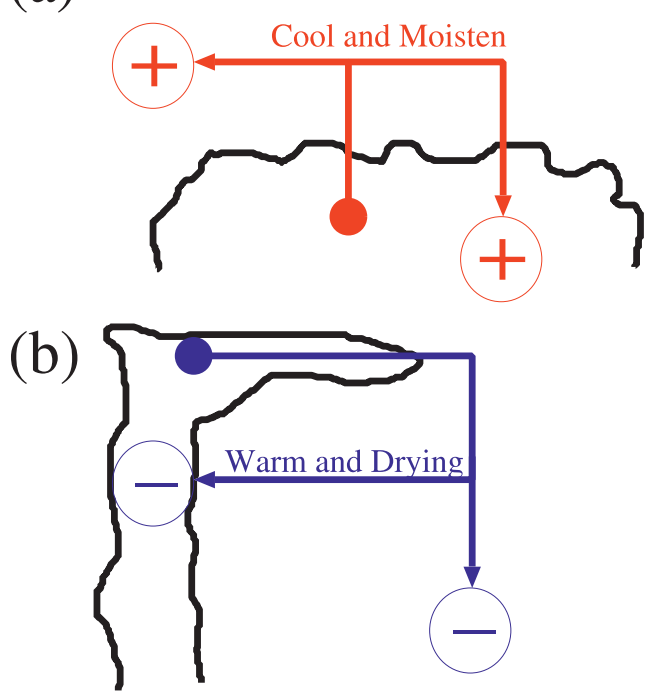

(c)

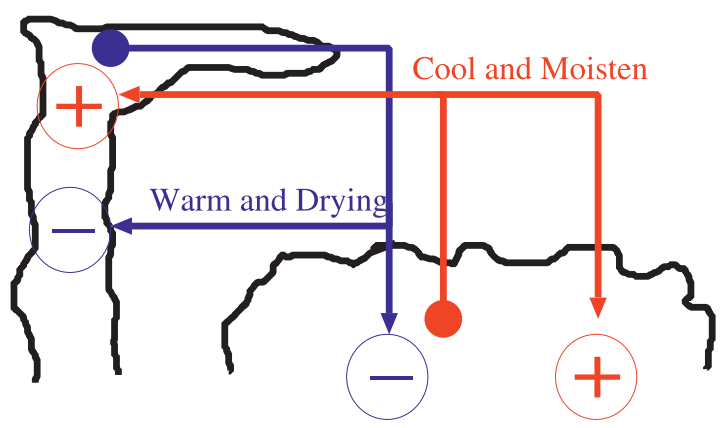

FIG. 1. Feedback loops for (a) shallow and (b) deep convection when they operate independently, and (c) when they are coupled. The arrows indicate the directions of feedbacks, and the plus and minus signs indicate positive and negative feedbacks, respectively. Here, feedbacks are defined in terms of the tendency for an increase of the cloud work function. Thus, positive and negative feedbacks lead to destabilization and stabilization of the system, respectively. In (a) shallow convection brings a positive feedback into the system, destabilizing itself, and also the conditions for deep convection by moistening and cooling the environment. In (b) deep convection brings a negative feedback into the system, stabilizing itself, and also the conditions for shallow convection by drying and warming the environment. In (c) the coupling of shallow and deep convection leads to a stable configuration by balancing the destabilization and stabilization tendencies (positive and negative feedbacks) of shallow and deep convection, respectively.

$A_{s}$ and $A_{d}$ for both shallow and deep convection, as indicated by arrows and a positive sign in Fig. 1a. Thus, $\mathcal{K}_{i, s}>0$ in Eq. (4) with $i=s, d$.

A destabilization tendency is also associated with a mechanism known as cloud-top entrainment instability (CTEI; Deardorff 1980; Randall 1980) that has been proposed as a trigger for the transformation of stratocumulus into trade cumulus convection. The mechanism destabilizes the existing clouds (Holland and Rasmusson 
1973, Nitta and Esbensen 1974) and is sometimes associated with deepening of stratiform clouds (Stevens et al. 2003). Reevaporation of cloud water is a robust and important process, because this is the only possible way to close the water cycle for nonprecipitating clouds.

In contrast, for deep convection, most of the condensed water is precipitated without evaporation. The dominant action of deep convection is warming of the environment by diabatic thermal compression of descending air in compensation against convective ascent. Warming stabilizes the atmosphere, which is manifest as a negative feedback that decreases the cloud work functions for both shallow and deep convection. Thus, $\mathcal{K}_{i, d}<0$ in Eq. (4) with $i=s, d$. This tendency is indicated by arrows and a negative sign in Fig. 1b. The process is called moist convective damping (Emanuel et al. 1994) and is also schematically shown by Fig. 11 of Arakawa and Schubert (1974).

The contrasting actions of shallow and deep convection on the environmental state can be incorporated into the cloud work function tendency equation (4) as follows:

$$
\begin{aligned}
& \frac{d A_{s}}{d t}=\mu_{s} M_{B, s}-\gamma_{s} M_{B, d} \\
& \frac{d A_{d}}{d t}=\mu_{d} M_{B, s}-\gamma_{d} M_{B, d} .
\end{aligned}
$$

Here $\mu$ and $\gamma$ are positive coefficients describing the destabilization and stabilization tendencies by shallow and deep convection, respectively, consistent with the sign of the matrix elements $\mathcal{K}_{i, j}$ just discussed. These coefficients can be evaluated for each cloud type from the environmental profile using formulas given in appendix B of Arakawa and Schubert (1974). Their Figs. 11 and 12, referred to above, illustrate the physical meaning of these coefficients. In following this physical picture, the coefficients are assumed to be constant for the present conceptual demonstration.

The system, composed of Eqs. (3) and (5), both for shallow and deep convection, can be closed by assuming that the convective kinetic energy is proportional to the cloud-base mass flux (Yano and Plant 2012), as shown:

$$
K=\alpha M_{B}
$$

with a constant $\alpha$. The assumption may be justified from explicit convection modeling studies (Emanuel and Bister 1996; Shutts and Gray 1999; Parodi and Emanuel 2009). The precise form of this assumption is not essential for the qualitative behavior of the coupled system to be discussed below. We obtained broadly similar results by following an alternative assumption (Pan and Randall 1998), as can be checked analytically. Under the formulation of Eqs. (3), (5), and (6), first we show how each type of convection evolves in isolation, and then we show an effect of coupling the two types together. Model parameters and numerical methods are briefly described in appendix A separately.

\section{Results}

Figure 2a shows the evolution of the shallow type in isolation: evaporative cooling keeps increasing the cloud work function (red), which in turn generates more kinetic energy (blue), leading to an explosion of shallow convection within a few days. A linear analysis, given separately in appendix B, shows that the initial cloud work function must be above the threshold $\alpha_{s} / \tau_{s}$ in order to obtain such an explosive growth. Appendix A presents an estimate of a typical value for the threshold cloud work function, which is relatively low at $2 \mathrm{~J} \mathrm{~kg}^{-1}$. Thus, although shallow convection can be dissipated away under a low cloud work function state, if it attains a shallow cloud work function above the threshold, then its destabilization tendency wins out over the dissipation. In more realistic situations, shallow convection has an equilibrium solution under a large-scale descent (i.e., a negative large-scale forcing, $F_{s}<0$ ). However, as also shown in appendix B, such an equilibrium solution is linearly unstable, and so the shallow-only system rather easily produces a runaway process.

The analysis of the shallow-convection-only case leads to rather unrealistic results because it is artificially assumed that only shallow convection can exist in the system, and that it does not interact with other convective modes. However artificial such an assumption may be, we should also realize that it expresses a real danger of artificial behavior that may arise in numerical models when a more realistic shallow convection parameterization is constructed without taking explicit account of the interactions with deep convection.

In contrast, Fig. $2 \mathrm{~b}$ shows the evolution of the deep type in isolation: deep convective kinetic energy (black) is initially enhanced by consuming the initial cloud work function (green), but then it simply damps out in less than 1 day due to its self-stabilization tendency (Emanuel et al. 1994). The result suggests that deep convection can only be sustained under the support of another process. In particular, when a positive fixed large-scale forcing is added, the system with deep convection alone attains a stable periodic cycle, as Yano and Plant (2012) have shown.

The above-mentioned two examples, although they may look unbelievably oversimplified, serve to demonstrate 

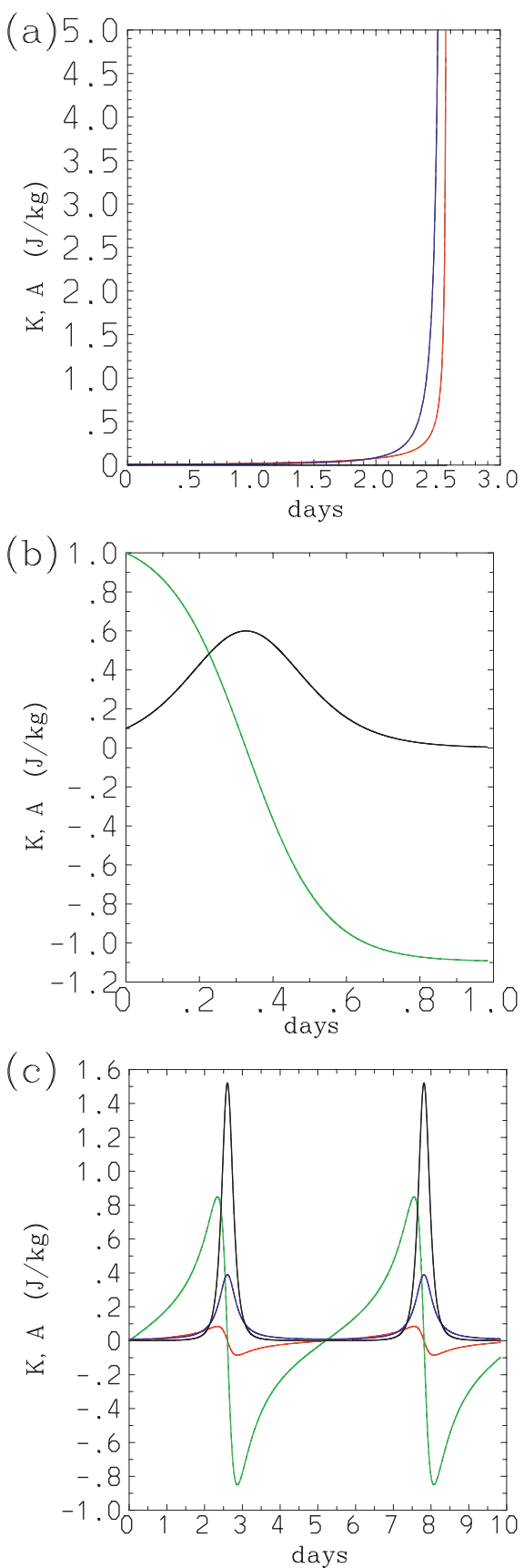

FIG. 2. Characteristic evolution of the atmospheric convective system when (a) shallow nonprecipitating convection alone is considered to be active, (b) deep precipitating convection alone is considered to be active, and (c) shallow and deep convection are coupled. The curves show $K_{s}$ (blue), $A_{s}^{\prime}$ (red), $K_{d}$ (black), and $A_{d}^{\prime}$ (green). Note that only the anomalous, nonequilibrium part of the cloud work functions are shown, with $A_{s}^{\prime}=A_{s}-\left(\alpha_{s} / \tau_{s}\right)$ and $A_{d}^{\prime}=A_{d}-\left(\alpha_{d} / \tau_{d}\right)$. Note also that the kinetic energies $K_{s}$ and $K_{d}$ are presented in the unit of $\mathrm{J} \mathrm{kg}^{-1}$ by dividing them by $10^{3} \mathrm{~kg} \mathrm{~m}^{-2}$ and $10^{4} \mathrm{~kg} \mathrm{~m}^{-2}$, respectively. The rescalings roughly correspond to the air mass for the depths 1 and $10 \mathrm{~km}$, respectively. Keep in mind that these kinetic energies are defined as an average over the whole grid box, and that they contain a factor $\sigma_{c}$ as part of their definition. the basic characteristics of the two convective types when considered strictly in isolation. We, again, emphasize that although the demonstrative setting itself is oversimplified, the energy cycle formulation on which our demonstration is based is robust and general. Thus, the demonstrated tendency must be there also in more realistic situations, although it may be less visible in the presence of many other processes.

As the main implication, when a global model deals with each type separately, using a different parameterization scheme for each, it may have difficulties in controlling shallow convection, and in generating deep convection. We speculate that some unphysical tunings may have arisen within operational models in order to overcome these problems, and that such tunings may have introduced other problems of their own: possible issues include systematic underestimations of marine boundary layer clouds (Zhang et al. 2005) as well as their cloud-albedo feedback (Bony and Dufresne 2005), and difficulties in successfully simulating large-scale organized structures associated with deep convection (Lin et al. 2006), such as the Madden-Julian oscillation (MJO).

An explicit coupling of the two types of convection can overcome these difficulties (Fig. 1c). In the twomode system, shallow convection's positive feedback is indispensable for engendering deep convection, and its continued presence is essential for sustaining self-damping deep convection. In turn, the negative feedback from deep convection is required for curbing self-explosive shallow convection. An example of the simultaneous evolution of the two types of convection under a coupled configuration is shown in Fig. 2c: a periodic cycle is realized. For the first 2 days, only shallow convection (blue) is apparent, which leads to gradual growth of deep convection (black). However, once deep convection kicks in, it rapidly dominates over shallow convection and leads to suppression of the latter. The suppression of shallow convection also suppresses deep convection, closing a cycle.

In the example shown, the oscillation period is around 5 days, but this can be made longer or shorter by taking the initial values of the convective kinetic energies to be smaller or larger, respectively. To realize a periodic state, the couplings must be maintained at an appropriate level so that neither the explosion of shallow convection nor the damping of deep convection dominates.

In the real atmosphere, planetary-scale circulations further help to regulate the convective system: under descending large-scale conditions, shallow convection can be maintained with less deep convection, as observationally known, and under ascending large-scale conditions, deep convection is maintained with less shallow convection. Thus, given suitable couplings, atmospheric 
convection may be maintained at self-organized criticality irrespective of large-scale conditions, as suggested by observations (Yano et al. 2001; Peters and Neelin 2006).

As already emphasized by Yano and Plant (2012), coupling of the present energy cycle model with the large-scale circulation is relatively straightforwardthat would lead to a model similar to those of Mapes (2000), Majda and Shefter (2001), Fuchs and Raymond (2005, 2007), Raymond and Fuchs (2007), and others, but offering the prospect of more robust and physically defensible closure hypotheses. Such a coupling to the large scale under the present theoretical framework is likely to lead to rich morphologies of convectively coupled equatorial waves, and so is left for future studies.

\section{Discussion}

The convective energy cycle formulation of Eqs. (3) and (5), adopted for the present study, can formally be derived under the mass flux convection parameterization framework as outlined in section 2. Thus, the formulation could be implemented within a standard mass flux parameterization, and indeed a similar energy cycle was implemented into a spectral parameterization by Pan and Randall (1998). A limitation of their implementation is that off-diagonal terms, $\mathcal{K}_{i, j}(i \neq j)$ in Eq. (4), were neglected so that interactions between different convection modes were excluded. Here, we propose that such interactions should be accounted for by retaining the offdiagonal terms.

The convective energy cycle furthermore provides a valuable framework for analyzing explicit convection simulations (cf. Yano et al. 2005a). Such a theoretically solid framework is clearly required for better understanding of the processes simulated by both cloudresolving and large-eddy-simulation models. This need is becoming increasingly more acute as simulations with higher resolutions and larger domains are performed (e.g., Khairoutdinov and Randall 2006).

Most current global models split the parameterization of convection into separate "bulk" descriptions of shallow and deep convection (Tiedtke 1989; Plant 2010). Here, we have considered the self-interactions of convective clouds in terms of a bulk pair system, interpreting the pair as being a severe truncation of the full convection spectrum into those two main types. An explicit coupling of the types is likely to lead to better simulation of various processes that are sensitive to the interactions between shallow and deep convection: examples are the diurnal cycle of convection (Guichard et al. 2004), tropical cyclogenesis (Emanuel 1989), and the slow recovery of tropical convection in the aftermath of a dry intrusion (Parsons et al. 2000). Benedict and
Randall (2007) emphasize the importance of the transformation process from shallow to deep convection in the MJO cycle.

Clearly, the coupled equation set used here has been simplified in order to make a particular point, as already emphasized in various places. We could, for example, point to the importance of midlevel clouds (cumulus congestus) as a third separate category that mediates the transformation to deep convection in some situations (Johnson et al. 1999). The presentation here also neglects the role of downdrafts (Zipser 1969, 1977) as well as the tendency of convection to organize on mesoscales and beyond (Moncrieff 2010). Clearly, there are rich morphologies of atmospheric convection that could be incorporated. These various additional physical elements can relatively easily be included into the present energy cycle formulation by applying the mode-decomposition principle (Yano et al. 2005b).

It should also be recalled that the standard mass flux formulation leading to this energy cycle system does not consider direct interactions between convective types, but rather describes interactions as mediated by the environment. The mode-decomposition framework proposed by Yano et al. (2005b; see also Yano et al. 2010; Yano 2011) does, however, provide a basis for taking direct interactions into account.

In conclusion, we nevertheless emphasize that the complexities of the relationship between shallow and deep convection become strikingly simple when expressed by a coupled energy cycle model. The coexistence of many relevant processes should not obscure this basic point. We also emphasize that this relatively straightforward coupling of the shallow and deep convection schemes under a fully prognostic closure based on the convective energy cycle is both a practical and important objective for model development.

Acknowledgments. This work was supported by a joint project award from the Royal Society and CNRS, and performed under a framework of COST Action ES0905. Discussions with Bjorn Stevens are acknowledged.

\section{APPENDIX A}

\section{Model Parameters}

Based on our earlier estimates for deep convection (Yano and Plant 2012), and on a similar process of estimation for the corresponding vertical integrals relevant for shallow convection, we choose the physical parameters to be $\mu_{s}=\gamma_{s}=0.1 \mathrm{~J} \mathrm{~m}^{2} \mathrm{~kg}^{-1}, \mu_{d}=\gamma_{d}=$ $1 \mathrm{~J} \mathrm{~m}^{2} \mathrm{~kg}^{-1}, \alpha_{s}=2 \times 10^{3} \mathrm{~m}^{2} \mathrm{~s}^{-1}, \alpha_{d}=1 \times 10^{4} \mathrm{~m}^{2} \mathrm{~s}^{-1}$, and $\tau_{s}=\tau_{d}=10^{3} \mathrm{~s}$. Note that the above-mentioned 
parameters lead to equilibrium (threshold) values for the cloud work functions $A_{s}=\alpha_{s} / \tau_{s}=2 \mathrm{~J} \mathrm{~kg}^{-1}$ and $A_{d}=$ $\alpha_{d} / \tau_{d}=10 \mathrm{~J} \mathrm{~kg}^{-1}$ (cf. appendix B). These values are small in comparison with typical values for CAPE, but they are comparable with available observational estimates of the cloud work function (Lord and Arakawa 1980).

We initialize the convective kinetic energy to have values of $K_{s}=10 \mathrm{~J} \mathrm{~m}^{-2}$ and $K_{d}=0 \mathrm{~J} \mathrm{~m}^{-2}$ for Fig. 2a, $K_{s}=0 \mathrm{~J} \mathrm{~m}^{-2}$ and $K_{d}=10^{3} \mathrm{~J} \mathrm{~m}^{-2}$ for Fig. 2b, and $K_{s}=$ $K_{d}=10 \mathrm{~J} \mathrm{~m}^{-2}$ for Fig. 2c. All of the runs are initialized with the equilibrium (threshold) values of the cloud work functions listed above, except for the case of the deep cloud work function in Fig. 2b, which is initialized with an anomaly $A_{d}^{\prime}=1 \mathrm{~J} \mathrm{~kg}^{-1}$. An anomaly is included in that case because otherwise the initially weak deep convection simply dies out with time. Note that a weak but nonvanishing convective kinetic energy is required to ensure the later enhancement of any given convection type (Yano and Plant 2012).

See Yano and Plant (2012) for a detailed derivation of the energy cycle system considered herein. Numerical integrations are performed with the fourth-order RungeKutta method.

\section{APPENDIX B}

\section{Linear Stability Analysis of the Shallow Convection System}

The purpose of the present appendix is to analyze a system with shallow convection only, following the analysis for a system with deep convection only presented in Yano and Plant (2012), but in a more succinct manner. The system is given by Eqs. (3) and (5a). After substitution of the hypothesis (6) and neglecting deep convection, these equations reduce to

$$
\begin{aligned}
\frac{d A_{s}}{d t} & =\mu_{s} M_{B, s} \\
\frac{d M_{B, s}}{d t} & =\left(\frac{A_{s}}{\alpha_{s}}-\frac{1}{\tau_{s}}\right) M_{B, s} .
\end{aligned}
$$

An equilibrium solution of the system is given by $M_{B, s}=0$ with an arbitrary value for the cloud work function, say, $\bar{A}_{s}$. From Eq. (B1b), a linear perturbation about this equilibrium state evolves as

$$
\frac{d M_{B, s}^{\prime}}{d t}=\left(\frac{\bar{A}_{s}}{\alpha_{s}}-\frac{1}{\tau_{s}}\right) M_{B, s}^{\prime},
$$

with the prime denoting a perturbation. The equation shows that perturbations are damped if the cloud work function is below the threshold $\bar{A}_{s}<\bar{A}_{s}^{\text {eq }} \equiv \alpha_{s} / \tau_{s}$ and exponentially growing above the threshold $\bar{A}_{s}>\bar{A}_{s}^{\text {eq }}$. The equilibrium solution is, thus, stable only if the cloud work function is below the threshold so that dissipation wins out over the self-destabilization tendency.

If the cloud work function exceeds the threshold, then a mass flux perturbation grows exponentially, and it further leads to exponential growth of the cloud work function, according to Eq. (B1a). It is important to note that the threshold value, $\bar{A}_{s}^{\mathrm{eq}}=2 \mathrm{~J} \mathrm{~kg}^{-1}$ estimated in appendix A, is relatively small and easily exceeded in typical environmental states for which stratocumulus and trade wind clouds are dominant. Thus, shallow convection in isolation is often self-destabilizing as discussed in the main text.

Large-scale descent can be incorporated into the system by means of a negative value for the large-scale forcing, $F_{s}<0$, so that Eq. (B1a) reads

$$
\frac{d A_{s}}{d t}=\mu_{s} M_{B, s}+F_{s},
$$

which has an equilibrium solution with finite shallow convection:

$$
M_{B, s}=M_{B, s}^{\mathrm{eq}} \equiv-F_{s} / \mu_{s}
$$

In this case, the threshold $\bar{A}_{s}^{\text {eq }}$, defined above, is the unique equilibrium value for the cloud work function. However, this equilibrium solution is unstable against any linear perturbation, as can immediately be seen by inspection of the linearized perturbation equations

$$
\begin{gathered}
\frac{d A_{s}^{\prime}}{d t}=\mu_{s} M_{B, s}^{\prime}, \\
\frac{d M_{B, s}^{\prime}}{d t}=\frac{M_{B, s}^{\mathrm{eq}}}{\alpha_{s}} A_{s}^{\prime} .
\end{gathered}
$$

\section{REFERENCES}

Arakawa, A., and W. H. Schubert, 1974: Interaction of a cumulus cloud ensemble with the large-scale environment, Part I. J. Atmos. Sci., 31, 674-701.

Benedict, J. J., and D. A. Randall, 2007: Observed characteristics of the MJO relative to maximum rainfall. J. Atmos. Sci., 64, 2332-2354.

Bony, S., and J.-L. Dufresne, 2005: Marine boundary layer clouds at the heart of tropical cloud feedback uncertainties in climate models. Geophys. Res. Lett., 32, L20806, doi:10.1029/ 2005 GL023851.

Bretherton, C. S., and Coauthors, 2004: The EPIC 2001 stratocumulus study. Bull. Amer. Meteor. Soc., 85, 967-977.

Deardorff, J. W., 1980: Cloud top entrainment instability. J. Atmos. Sci., 37, 1211-1213. 
Emanuel, K. A., 1989: The finite amplitude nature of tropical cyclogenesis. J. Atmos. Sci., 46, 3431-3456.

_- and M. Bister, 1996: Moist convective velocity and buoyancy scales. J. Atmos. Sci., 53, 3276-3285.

— J. D. Neelin, and C. S. Bretherton, 1994: On large-scale circulation in convective atmospheres. Quart. J. Roy. Meteor. Soc., 120, 1111-1143.

Fuchs, Z., and D. J. Raymond, 2005: Large-scale modes in a rotating atmosphere with radiative-convective instability and WISHE. J. Atmos. Sci., 62, 4084-4094.

— disturbances with a humidity closure. Tellus, 59A, 344-354.

Guichard, F., and Coauthors, 2004: Modelling the diurnal cycle of deep precipitating convection over land with cloud-resolving models and single-column models. Quart. J. Roy. Meteor. Soc., 130, 3139-3172.

Holland, J. Z., and E. M. Rasmusson, 1973: Measurements of the atmospheric mass, energy, and momentum budgets over a 500-km square of tropical ocean. Mon. Wea. Rev., 101, 44-55.

Johnson, R. H., T. M. Rickenbach, S. A. Rutledge, P. E. Ciesielski, and W. H. Schubert, 1999: Trimodal characteristics of tropical convection. J. Climate, 12, 2397-2418.

Khairoutdinov, M., and D. Randall, 2006: High-resolution simulation of shallow-to-deep convection transition over land. J. Atmos. Sci., 63, 3421-3436.

Lin, J.-L., and Coauthors, 2006: Tropical intraseasonal variability in 14 IPCC AR4 climate models. Part I: Convective signals. J. Climate, 13, 2665-2690.

Lord, S. J., and A. Arakawa, 1980: Interaction of a cumulus cloud ensemble with the large-scale environment. Part II. J. Atmos. Sci., 37, 2677-2692.

Majda, A. J., and M. G. Shefter, 2001: Models for stratiform instability and convectively coupled waves. J. Atmos. Sci., 58, $1567-1584$

Mapes, B. E., 2000: Convective inhibition, subgrid-scale triggering energy, and stratiform instability in a toy tropical wave model. J. Atmos. Sci., 57, 1515-1535.

Moeng, C.-H., 1998: Stratocumulus-topped atmospheric planetary boundary layer. Buoyant Convection in Geophysical Flows, E. J. Plate, Eds., Kulwer Academic, 421-440.

Moncrieff, M. W., 2010: The multiscale organization of convection at the interaction of weather and climate. Why Climate Vary? Geophys. Monogr., Vol. 189, Amer. Geophys. Union, $3-26$.

Nitta, T., and S. Esbensen, 1974: Heat and moisture budget analyses using BOMEX data. Mon. Wea. Rev., 102, 17-28.

Pan, D.-M., and D. A. Randall, 1998: A cumulus parameterization with prognostic closure. Quart. J. Roy. Meteor. Soc., 124, 949981.

Parodi, A., and K. Emanuel, 2009: A theory for buoyancy and velocity scales in deep moist convection. J. Atmos. Sci., 66, 3449-3463.

Parsons, D. B., K. Yoneyama, and J.-L. Redelsperger, 2000: The evolution of the tropical western Pacific atmosphere-ocean system following the arrival of a dry intrusion. Quart. J. Roy. Meteor. Soc., 126, 517-548.
Peters, O., and J. D. Neelin, 2006: Critical phenomena in atmospheric precipitation. Nat. Phys., 2, 393-396.

Plant, R. S., 2010: A review of the theoretical basis for bulk mass flux convective parameterization. Atmos. Chem. Phys., 10, 3529-3544.

Randall, D. A., 1980: Conditional instability of the first kind upside-down. J. Atmos. Sci., 37, 125-130.

- and Coauthors, 2007: Climate models and their evaluation. Climate Change 2007: The Physical Basis, S. Solomon et al., Eds., Cambridge University Press, 589-662.

Raymond, D. J., and Z. Fuchs, 2007: Convectively coupled gravity and moisture modes in a simple atmospheric model. Tellus, 59A, 627-640.

Shutts, G. J., and M. E. B. Gray, 1999: Numerical simulations of convective equilibrium under prescribed forcing. Quart. J. Roy. Meteor. Soc., 125, 2767-2787.

Stevens, B., 2005: Atmospheric moist convection. Annu. Rev. Earth Planet. Sci., 33, 605-643.

— , and Coauthors, 2003: On entrainment rates in nocturnal marine stratocumulus. Quart. J. Roy. Meteor. Soc., 129, 34693493.

Tiedtke, M., 1989: A comprehensive mass flux scheme of cumulus parameterization in large-scale models. Mon. Wea. Rev., 117, 1779-1800.

Wu, C. M., B. Stevens, and A. Arakawa, 2009: What controls the transition from shallow to deep convection? J. Atmos. Sci., 66, 1793-1806.

Yano, J.-I., 2011: Mass-flux subgrid-scale parameterization in analogy with multi-component flows: A formulation towards scale independence. Geosci. Model Dev. Discuss., 4, $3127-$ 3160 .

— equilibrium: Periodic cycle and discharge-recharge mechanism. Quart. J. Roy. Meteor. Soc., 138, 626-637.

_, K. Fraedrich, and R. Blender, 2001: Tropical convective variability as $1 / f$-noise. J. Climate, 14, 3608-3616.

— J.-P. Chaboureau, and F. Guichard, 2005a: A generalization of CAPE into potential-energy convertibility. Quart. J. Roy. Meteor. Soc., 131, 861-875.

—, J.-L. Redelsperger, F. Guichard, and P. Bechtold, 2005b: Mode decomposition as a methodology for developing convective-scale representations in global models. Quart. J. Roy. Meteor. Soc., 131, 2313-2336.

—, P. Bénard, F. Couvreux, and A. Lahellec, 2010: NAM-SCA: A nonhydrostatic anelastic model with segmentally constant approximations. Mon. Wea. Rev., 138, 1957-1974.

Zhang, M. H., and Coauthors, 2005: Comparing clouds and their seasonal variations in 10 atmospheric general circulation models with satellite measurements. J. Geophys. Res., 110, D15S02, doi:10.1029/2004JD005021.

Zipser, E. J., 1969: The role of organized unsaturated convective downdrafts in the structure and rapid decay of an equatorial disturbance. J. Appl. Meteor., 8, 799-814.

_- 1977: Mesoscale and convective-scale downdrafts as distinct components of squall-line structure. Mon. Wea. Rev., 105, 1568-1589. 